\title{
17. El pluscuamperfecto en las lenguas románicas
}

Ingmar Söhrman

Göteborgs universitet

\section{Introducción}

Se suele considerar el pluscuamperfecto un tiempo gramatical sin complicaciones que describe una acción anterior a otra en el pasado. Resulta que esta idea es solo parcialmente correcta. Este tiempo tiene otras funciones sintácticas y modales.

En cuanto a la tipología podemos constatar que existen muchas semejanzas entre las lenguas románicas tanto en lo formal como en lo funcional. Intentaremos comparar las formas y los usos de los idiomas románicos con los del latín.

Casi todas las lenguas románicas (con la excepción del rumano y, en realidad, del gallego) han desarrollado un pluscuamperfecto analítico o perifrástico, basado en el auxiliar latino habere y en ciertas lenguas occidentales y con un sentido especial también en tenere o además en essere (latín tardío) < esse más el participio pasado, y la mayoría de estos idiomas han perdido el sintético, que era el único en latín clásico. Veremos que también la idea del pluscuamperfecto como un tipo de pretérito imperfecto pasado (acontecimientos estáticos y procesales) no es completamente correcta, ya que se usa para acciones perfectivas también (Westerholm 2010: 94-I04); además, queda evidente que no es necesaria la relación con otro tiempo del pasado, sino que, a veces, se utiliza para expresar sucesos resultativos que se refieren directamente al presente en el momento de la enunciación.

Pocos lingüistas se han dado cuenta del hecho de que este tiempo también puede expresar modalidades de diferentes tipos que pueden

Cómo citar este capítulo:

Söhrman, Ingmar, El pluscuamperfecto en las lenguas románicas. In: Engwall, Gunnel \& Fant, Lars (eds.) Festival Romanistica. Contribuciones lingüísticas - Contributions linguistiques - Contributi linguistici - Contribuições linguísticas. Stockholm Studies in Romance Languages. Stockholm: Stockholm University Press. 201 5, pp. 334-345. DOI: http://dx.doi.org/IO.I6993/bac.q. License: CC-BY 
resumirse en dos "conceptos"-cambio del mundo referencial y aumento de la fuerza ilocutoria modificativa.

En el presente trabajo intentamos aclarar las formas y usos del pluscuamperfecto de forma coherente y holística incluyendo todas las lenguas románicas y con perspectivas tanto sincrónicas como diacrónicas (cf. Söhrman 2013).

\section{Formas}

El portugués nos da ejemplos de los tres tipos formales que se usan en las lenguas románicas para expresar la función del pluscuamperfecto (ej. I-3).

(I) Samuel aproximou-se para avisar que o táxi tinha chegado. (C. Drummond de Andrade I984: I30)

(2) Samuel aproximou-se para avisar que o táxi havia chegado.

(3) Samuel aproximou-se para avisar que o táxi chegara.

En el primer ejemplo tenemos el auxiliar latino tenere (port. ter) + participio pasado (en adelante pp) y en el segundo habere (haver) + participio pasado, mientras que el tercer ejemplo es el descendiente directo del pluscuamperfecto latino plicaveram 'había plegado'. En portugués es el primer tipo que es el más usado, y a los otros dos se los considera más literarios, y prácticamente solo usados en el lenguaje escrito. Así, desde una perspectiva portuguesa es curioso que sea el segundo tipo (habere $+\mathrm{pp}$ ) el más usado en la mayoría de las lenguas románicas. No obstante, estas se diferencian del gallego que en realidad solo usa el tipo del tercer ejemplo (-ra) y el rumano que no tiene otras formas que las de este último tipo. No obstante, hace falta reconocer que es el pluscuamperfecto del subjuntivo latino el que ha tomado el papel general del pluscuamperfecto rumano, es decir, lat. captavissem 'captar; anhelar' > rum. cautasem 'yo había buscado'. Además es un tiempo que está restringido al lenguaje escrito. En el lenguaje hablado el perfecto perifrástico lo ha sustituido a cautat 'ha buscado' (Söhrman 2009).

En español queda el uso de las formas en -ra, sobre todo en el lenguaje escrito (periodístico) con la función del pluscuamperfecto del indicativo en subordinadas relativas (Hermerén I992; cf. ej. 4), aunque su función normal es la del imperfecto del subjuntivo. 
(4) El señor López, que fuera director del instituto, murió ayer.

Posner (I996: I37) también sugiere el uso de estas formas en el español andino para expresar cierta distancia mental a la acción (cf. la distancia cognitiva discutida abajo):

In the Spanish of the Andes the past tense of the compound form (pluperfect) seems to imply lack of personal commitment to the statement made, rather than past timing.

Da el ejemplo boliviano "y en nada habían encontrado trabajo" donde se usaría el perfecto en el español estándar "y en nada han encontrado trabajo". También puede usarse en lugar del presente del indicativo en expresiones como "había sido tarde" donde se esperaría "ya es tarde" (Bosque 2009: I789).

Antes de comparar las diferentes construcciones, hace falta notar que ciertas lenguas románicas, al igual que el alemán, usan el verbo essere como auxiliar como ya hemos constatado. Este uso se limita al centro de Romania, es decir al francés, occitano, retorromance e italiano. El uso de essere está reducido a verbos intransitivos de movimiento como fr. Je suis venu, it. sono venuto, pero en estos casos se diferencian el género y el número -cf. fr. Je suis venue, it. sono venuta en femenino y en el plural femenino fr. nous sommes venues, it. siamo venute.

En gallego se usa normalmente, como ya hemos constatado, la construcción analítica, y la perifrástica con el auxiliar haber está restringida a oraciones impersonales (cf. 5; Álvarez et al. I986: 4I4). La construcción tiene también un sentido resultativo en catalán y en español, aunque el uso del auxiliar tenere + pp es muy poco frecuente (ej. 6) en estas lenguas.

(5) Naquel transatlântico había embarcadas unhas cincocentas persoas. (Álvarez et al. I986:4I4)

(6) Tenía escritas todas las cartas.

En el latín clásico se encuentran también construcciones perifrásticas como habeo/teneo comparatum cultellum 'había comprado el cuchillo' (Elcock I960: I2I); Durante (I98I: 27-29) indica que ya en el primer siglo DC el uso del pluscuamperfecto era menos frecuente en latín. 
Y ahora resumimos las formas posibles del pluscuamperfecto en las lenguas románicas:

Tabla 1. El pluscuamperfecto en diversas lenguas románicas

\begin{tabular}{lll}
\hline Lenguas & Formas perifrásticas & Formas sintéticas \\
\hline Portugués & tinha/havia $+\mathrm{pp}$ & falara \\
Galego & (había $+\mathrm{pp}$ ) & falara \\
Español & había $+\mathrm{pp}$ & hablara \\
Catalán & havia $+\mathrm{pp}$ & - \\
Occitano & aviéu/ère $+\mathrm{pp}$ & - \\
Francés & j'avais/j'étais $+\mathrm{pp}$ & - \\
Sursilvano- & jeu [ha]vevel/ erel + pp & - \\
(Vallader) & (eu vaiva/eira $+\mathrm{pp})$ & \\
Ladín dolomítico & ie ove/fove $+\mathrm{pp}$ & - \\
Italiano & avevo/ero $+\mathrm{pp}$ & - \\
Sardo & aío $+\mathrm{pp}$ & - \\
Rumanian & $-(\mathrm{am}+\mathrm{pp}=\mathrm{perfecto})$ & vorbiseram \\
\hline
\end{tabular}

Queda obvio que son las formas perifrásticas o analíticas las que predominan. Esta primera parte de nuestra exposición muestra la perspectiva formal del pluscuamperfecto.

\section{Funciones}

¿Cuáles son entonces las funciones de este tiempo? Según el diccionario Vox el pluscuamperfecto es "el pretérito cuya acción es anterior a otra también pretérita: había contado y hubiera o hubiese contado del indicativo y del subjuntivo, respect[ivamente].”

(7) Fr: J'avais acheté la voiture quand ma fille est née.

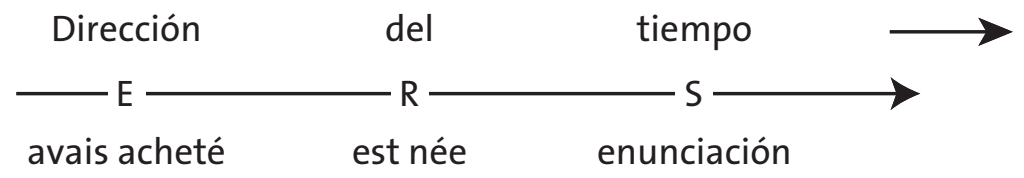

\section{Figura 1.}

Si seguimos el clásico modelo de Reichenbach (I980) tenemos los tres momentos de acción: el del acontecimiento (Point of event $=\mathrm{E}$ ), 
el de la referencia (Point of reference $=\mathrm{R}$ ) y el del habla o enunciación (Point of speech = S) (cf. Bermúdez Wachtmeister 2005: I69). En una oración como (7; Figura I arriba) se ve el modelo de Reichenbach E-R-S (cf. Bermúdez Wachtmeister 2005: I69).

Ya hace más de 80 años Jespersen (I93I) hizo hincapié en la complejidad del pluscuamperfecto y constató que este tiempo, en primer lugar, sirve para significar lo que pasó antes del tiempo del pasado o representa un pasado retrospectivo -dos cosas que están en la misma relación que el pretérito indefinido y el perfecto, pero son difíciles de separar (Jespersen I93 I:8I).

En un estudio sobre el pluscuamperfecto en griego clásico Krüger \& Cooper (1997) discuten el problema del aspecto del pluscuamperfecto griego, que entonces era sintético aunque se puede notar como surgieron las formas analíticas (Autor 20I3: I 8I). Sin embargo, parece que la constatación de Krüger \& Cooper también vale para el español y, por lo tanto, para las lenguas románicas:

The pluperfect expresses completion of verbal action in the past. But the idea of a past action to which the pluperfect relates is not necessarily called for. The pluperfect to an indefinite past time as the perfect relates to the idea, with all its imprecision, of an indefinite present time. In some ways the pluperfect is to the perfect as imperfect to present. But in verbal aspect there is a great difference. Both present and imperfect are properly durative and so at least potentially conative. The pluperfect shows an idea of completion much like that shown by the perfect, which is built up on the same stem. Yet the pluperfect is often associated with the imperfect and competes with and alternates with whenever its essential idea of fixed condition does not impede. So the pluperfect competes with the imperfect in description and particularization. The Latin pluperfect and English periphrases with had do not prepare very well for this tense [...] (1997: 632)

En su estudio sobre los tiempos del pasado en español y ruso Westerholm (2010: 94-I04) muestra claramente que, a menudo, se traduce el pluscuamperfecto español con el pasado perfectivo ruso y que éste frecuentemente corresponde al pluscuamperfecto español, lo que apoya las ideas de Krüger \& Cooper.

Es notable que cuando surgieron las lenguas románicas del latín vulgar el sistema verbal sintético, por lo menos parcialmente, se convirtiera en uno analítico. Varios lingüistas (Comrie I985, Bertinetto I986: 449, Haverling 2013) constatan la complejidad del tiempo y prefieren ver el pluscuamperfecto como un tiempo absoluto-relativo y no solo relativo como se solía decir antes, ya que tiene su uso "autónomo" y no 
solo relacionado a otras acciones. No es únicamente que el pluscuamperfecto refleje acciones perfectivas sino también resultativas y, como veremos más adelante, también modales.

Además, el pluscuamperfecto a veces solapa el uso del perfecto. Bertinetto muestra de forma convincente que es el aspecto perfectivo el que predomina en italiano aunque de ninguna manera excluye el aspecto imperfectivo (Bertinetto I986: 455-464). Bosque insiste en que el término correcto en español sería el ante-pretérito en lugar de ante-copretérito como se discute en la gramática de la Real Academia Española (Bosque 2009): "no se ancla [necesariamente] en un tiempo simultáneo a un tiempo anterior al momento del habla, sino directamente en un pasado" (Bosque 2009: I787). Entonces la opinión de Bertinetto coincide con la de Bosque y parece tener valor para todas las lenguas románicas.

Por consiguiente, desde un punto de vista aspectual, el modelo de Reichenbach parece demasiado simplista como diferencia entre diferentes tipos de acción, es decir, no distingue entre el pretérito indefinido y el imperfecto. Por eso, Gosselin (I996 y 2005) ha propuesto un modelo más elaborado. Insiste en los intervalos entre el momento ingresivo (comienzo) y el egresivo (final) de cada acción: el de la enunciación (OI, O2), el del proceso ( $\left.\mathrm{BI}_{1}, \mathrm{~B}_{2}\right)$ y el de la referencia (I, II). A estos agrega el intervalo circunstancial (ctI, ct2) que constituye el complemento temporal. Concretamos este modelo en la Figura 2:

"Luis escribió una novela."

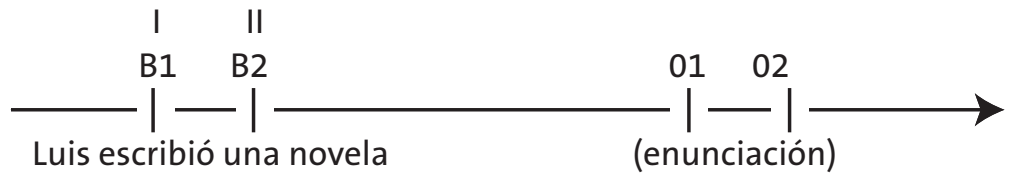

Figura 2.

Comparemos las correspondencias temporales de los tiempos de la Figura 2 con los de la Figura 3:

“(Cuando le vi) Luis escribía una novela."

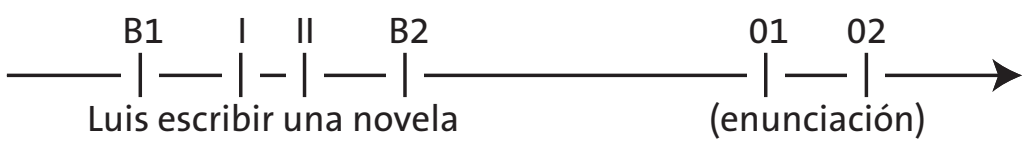

Figura 3. 
El intervalo del proceso, $\mathrm{B}_{\mathrm{I}}$ - B2, y el intervalo de referencia, I - II, no coinciden en la figura 3 como en la figura 2, lo cual da como resultado $\mathrm{E}=\mathrm{R}(-\mathrm{S})$ (cf. Reichenbach) en la figura 3, pero $\mathrm{E}<\mathrm{R}(-\mathrm{E})$, donde el signo < tiene su significado matemático (es decir "menos").

En sardo se usa a menudo el pluscuamperfecto en lugar del perfecto para describir acontecimientos sin relación con un punto de referencia en el presente o el pasado (cf. Blasco Ferrer I984: I 50). En el siguiente ejemplo (8), el contexto (cf. el complemento circunstancial temporal un'ora appustis 'una hora más tarde') nos enseña claramente que los sucesos descritos no están ubicados en un tiempo anterior al intervalo de referencia (Jones I993:85).

(8) Un'ora appustis l'aian muttiu chi esseret andau derettu derettu a domo sua.

Una hora más tarde le llamaron (habían llamado) para que fuera directamente a /su/ casa. (Jones I993:85-86).

Ménard (I987:I4I-I42) constata que en francés medieval tardío se usaba el pluscuamperfecto para marcar tanto la anterioridad como la duración, mientras que en el francés medieval antiguo se usaba el passé simple para todo sin preocuparse por la cronología relativa (cf. ej. 9).

(9) Dis blanches mules fist amener Marsilies que li tramist li reis de Suatilie (Chanson de Roland, 89).

Marsiles hizo traer diez mulas que le había dado el rey Suatilie.

Entonces, podemos ver que, desde una perspectiva románica, el pluscuamperfecto no tiene ninguna definición temporal clara, como la del pretérito, ya que es relativo a este tiempo (pretérito), aunque esta relación no siempre esté presente (cf. Westerholm 20I0: 47). Por lo tanto, queremos proponer el concepto de distancia cognitiva (o mental) como núcleo sintáctico del pluscuamperfecto, ya que la presencia de la deixis es la que da una función temporal al pluscuamperfecto mientras que la disminución de la deixis, dada por la falta de referencias temporales a otros sucesos, es lo que da un valor modal al pluscuamperfecto (Figura 4 ):

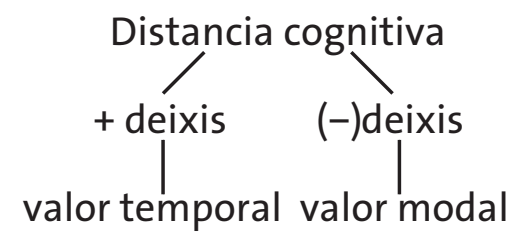

Figura 4. 
Con frecuencia la deixis se expresa a través de otra oración o frase como se puede ver en el ejemplo catalán (Io):

(Iо) A penes havia tirat la meva carta que vaig rebre la seva. Apenas había echado mi carta, recibí la suya.

\section{Valores modales}

En una oración como la del ejemplo (I I), vemos que la acción se conecta directamente con el momento de la enunciación.

(I I) Esta noche había ganado un millón de euros.

La referencia a esta noche incluye una implicatura conversacional que indica que lo que ocurrió fue un sueño y la distancia cognitiva en forma de un sueño se manifiesta y no necesita más indicaciones para que el oyente la perciba. En todos los ejemplos modales veremos que el pluscuamperfecto contiene un aspecto resultativo relativo al momento de la enunciación, lo que se puede ilustrar en el modelo de Gosselin de la siguiente manera, donde la flecha marca la relación directa con el momento de la enunciación:

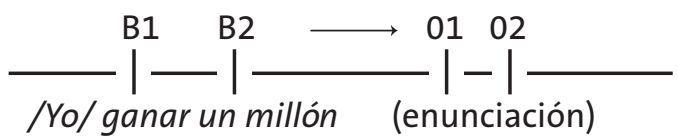

\section{Figura 5.}

Como constatamos ya al principio, se pueden dividir los usos modales en dos conceptos: Cambio del mundo referencial y Aumento de la fuerza ilocutoria modificativa, cada uno de los cuales puede dividirse en cuatro subgrupos:

Cambio del mundo referencial

I) Valor onírico (mundo soñado)

2) Valor fantástico (mundo inventado)

3) Valor irreal

4) Valor lúdico

Aumento de fuerza ilocutoria modificativa

I) Valor epistémico doxático (indica eventos conocidos o considerados como tales)

2) Valor de cortesía o modestia

3) Valor cariñoso

4) Valor de deducción narrativa 
Concretemos estas categorías con unos ejemplos clarificativos. Es interesante constatar que raras veces se usa el pluscuamperfecto con valor modal con verbos imperfectivos, sino que casi siempre se usa con perfectivos o resultativos.

Las cuatro subcategorías de cada concepto se diferencian, pero no solo limitan la una con la otra sino que se solapan y se pueden ver cómo las partes de un dardo de cuatro lados con una relación muy estrecha.

En la primera categoría Cambio del mundo referencial se nota claramente que la referencia no tiene ningún enlace con un momento del mundo real, sino con uno de otro mundo posible dentro del universo de creencias (univers de croyance; Martin I983: II8; Autor I99I: 4I-49). El hablante se refiere a un mundo ficticio donde rigen otras reglas e implica una situación de "Supongamos que...", pero no habla del pasado pero de algo cognitivamente lejano -probablemente alternativo- por no pertenecer a la realidad actual.

Con el valor onírico el hablante habla de algo soñado, y por lo tanto irreal, pero sin que el hablante haya podido influir en la realización del sueño (I 2) donde no es necesario incluir las palabras entre corchetes. Se podría incluir questa notte como indicación de un sueño, pero el contexto mismo muestra a qué tipo de mundo (real o posible) se refiere el hablante.

(I2) [Ho sognato che] avevo vinto un milione.

El valor fantástico indica un paso consciente a un mundo posible alternativo, inventado por el protagonista mismo ( $\mathrm{I}_{3}$ ) y se parece al onírico, pero representa una acción espontánea e inconsciente.

(I3) Et alors elle était devenue la grande star.

El valor irreal representa una especulación por parte del hablante sin que pase mentalmente a otro mundo como en el caso del valor fantástico (I3). Parece transformar la proposición en una condicional, pero se limita a imaginarse un mundo posible en el que no se entra (I4). El mensaje no es ninguna sorpresa para el emisor y la prótasis toma un valor concesivo en lugar de uno hipotético con la intención de hacer el mensaje más cortés.

(I4) Y si habian sido mozalbetes, ahora ya serían hombres y derechos.

El valor lúdico es un uso frecuente del lenguaje infantil donde compite con el potencial. El tiempo manda a los participantes a otro mundo 
posible (de ficción) -y ellos lo saben y lo admiten y pueden dejar esta "realidad imaginada" en cualquier momento según su propio deseo, pero el pluscuamperfecto tiene un valor performativo ( I 5 ).

(I 5) Io ero la mamma e te avevo appena preparato la cena.

Dentro del concepto Aumento de la fuerza ilocutoria modificativa, el pluscuamperfecto tiene una fuerza ilocutiva que modifica el mensaje, pero no es un traslado mental a otro mundo posible.

El valor epistémico doxático tiene un valor cortés, y los ejemplos contienen casi siempre una negación y se puede siempre añadir una «tag-question» (n'est-ce pas?/¿゙verdad?/non è vero?). Es una manera cortés de recordarle algo a alguien sin reducir la importancia del mensaje y, como veremos, es una manera eficaz de criticar a alguien con una insinuación formulada como pregunta.

(I6) Tu n’avais pas parlé avec Jean? [comme nous avions décidé hier]

El valor de cortesía o modestia sirve para recordar a alguien, sin molestarle y sin reproches; a diferencia del caso anterior que se usa "topdown"; este caso es "down-top", es decir recordando sin crítica desde una posición (psicológicamente) inferior -empleado a jefe, etc.

(I7) Avevo telefonato sta mattina.

El valor cariñoso es un valor pragmático (formulado como pregunta) que deja abierta al oyente la posibilidad de huir o protestar. Se nota el cariño claro del hablante (p.ej. madre a hijo) aunque lleve algo de crítica. Aquí la prosodia es fundamental para diferenciar esta categoría de las otras dos.

(I8) Tu n'avais pas mangé suffisamment?

La última subcategoría, el valor de deducción narrativa (I9), es una reducción de la responsabilidad enunciativa (Kronning 2003) que se produce en el momento de la enunciación, y es una deducción lógica, pero expresada con modestia. El valor epistémico queda claro y se acerca al valor de los verbos modales que pueden sustituir el pluscuamperfecto (20).

(19) Como el césped estaba húmedo, había llovido durante la noche.

(20) Como el césped estaba húmedo, debía de haber llovido durante la noche. 
Para concluir, solo necesitamos constatar que tanto en cuanto a la forma como en cuanto a la función, el pluscuamperfecto resulta más diferenciado de lo que se suele decir, que no es necesariamente un tiempo que se usa con verbos imperfectivos, sino que es igual de frecuente usarlo con verbos perfectivos y también puede representar un aspecto resultativo. Los valores modales son, por lo visto, muy notables en el lenguaje hablado, pero poco comentados.

\section{Referencias}

Álvarez. Rosario, Xosé Luis Reguiera \& Henrique Monteagudo. I986. Gramática Galega. Vigo: Galaxia.

Bermúdez Wachtmeister, Fernando. 2005. "Los tiempos verbales como marcadores evidenciales. El caso del pretérito perfecto compuesto". Estudios filológicos, 40. 165-193.

Bertinetto, Pier Marco. 1986. Tempo, aspetto e azione nel verbo italiano : il sistema dell'indicativo. Firenze: Accademia della Crusca.

Blasco Ferrer, Eduardo. I984. Storia della lingüística della Sardegna. Tübingen: Niemeyer.

Bosque, Ignacio et al (eds.). 2009. Nueva gramática de la lengua española, 2 vols. Real Academia española \& Asociación de Academias de la Lengua Española. Madrid: Espasa Libros.

Castillo Puche, José Luis. I976 [1956]. El vengador. Barcelona: Destino.

Comrie, Bernard. I985. Tense. Cambridge: Cambridge University Press.

Drummond de Andrade, Carlos. I984. Contos de Aprendiz, $22^{\text {nd }}$ ed. Rio de Janeiro: Ed. Record.

Durante, M. I98 I. Dal latino all'italiano moderno. Saggio di storia linguistica e culturale. Bologna: Zanichelli.

Elcock, William D. I97I [1960]. The Romance Languages. London: Faber \& Faber.

Gosselin, Lauren. I996. Sémantique de la temporalité en français. Louvain-laNeuve: Duculot.

Gosselin, Laurent. 2005. Temporalité et modalité. Bruxelles: de Boeck/Duculot.

Haverling, Gerd. 2013. "On tense and mood in conditional clauses from Early to Late Latin”. I3-56.In: Josephson, Folke \& Ingmar Söhrman (eds.)., Diachronic and Typological Perspectives on Verbs. Amsterdam: John Benjamins. 
Hermerén, Ingrid. I992. El uso de la forma en ra con valor no-subjuntivo en el español moderno. Études romanes de Lund 49. Lund: Lund University Press.

Horrock, Geoffrey. 2009. Greek: A History of the Language and its Speakers. London \& New York: Longman Linguistics Library.

Jespersen, Otto. I93I. A modern English grammar on historical principles. P. 4, Syntax, Vol. 3, time and tense. Germanische Bibliothek. Erste Reihe, Sprachwissenschaftliche Lehr- und Elementarbücher. Heidelberg: Winter.

Jones, Michael A. I993. Sardinian Syntax. London \& New York: Routledge.

Judge, Anne \& F. G. Healey. I983. A Reference Grammar of Modern French. London, Melbourne \& Baltimore: Edward Arnold.

Kronning, Hans 2003. "Modalité et évidentialité ». In: Birkelund, Merete, Gerhard Boysen \& Poul S. Kjærsgaard (eds.). Aspects de la Modalité. Tübingen: Max Niemeyer, Linguistische Arbeiten 469. I 3 I-I 5 I.

Krüger, Karl Wilhelm. I997. Attic Greek Prose Syntax. Vol. I. Guy L. Cooper III. (ed.). Ann Arbor: The University of Michigan Press.

Martin, Robert.1983. Pour une logique du sens. Paris: PUF. Horrocks, G. I997.

Ménard, Philippe. I987. Syntaxe de l'ancien français. Vol I. In: Lefèvre Yves (ed.). Manuel du français du Moyen Âge. Paris: Éd. Bière.

Posner, Rebecca. I996. The Romance languages. Cambridge: Cambridge University Press.

Reichenbach, Hans. I980 [1947]. Elements of Symbolic Logic. New York: Dover Publications.

Söhrman, Ingmar. I99I. Las construcciones condicionales en castellano contemporáneo. Studia Romanica Upsaliensia 48. Uppsala: Almqvist \& Wiksell

Söhrman, Ingmar. 2009. "Mai-mult-ca-perfectul în limba română într-o perspectivă romană ăi balcanică”. In: Kahl, Thede (ed.). Das Rumänische und seine Nachbaren. Leipzig: Frank \& Timme. I3 I-I39.

Söhrman, Ingmar. 20I3. "Reference, aspectuality and modality in ante-preterit (pluperfect) in Romance languages”. In:Josephson, Folke \& Ingmar Söhrman (eds.). Diachronic and Typological Perspectives on Verbs. Amsterdam: John Benjamins. I73-209.

Westerholm, David. 2010. Funciones del pasado en los sistemas verbales del español y del ruso. Acta Universitatis Gothoburgensis, Romanica Gothoburgensia 66. Göteborg: Göteborgs universitet.

Vox = Diccionario Enciclopédico Vox I. 2009, Larousse Editorial. 